\title{
Multi-Drug Resistance in Health Care-Associated Bacteremia in Intensive Care Units at King Fahad Specialized Hospital, Buraidah, Saudi Arabia
}

\author{
Khaled M. Hass ane in ${ }^{1}$, Marwa Salah Mostafa ${ }^{1, *}$, Raafat El- Sanhoty $^{2}$, K. M. Hass an ${ }^{3}$ \\ ${ }^{1}$ Department of Medical Microbiology \& Immunology, College of medicine, Qassim University, Buraidah, Saudi Arabia \\ ${ }^{2}$ Department of Food Science and Human Nutrition,, College of A griculture, Qassim University, Buraidah, Saudi Arabia \\ ${ }^{3}$ Department of Medical Laboratories, King Fahad Specialized Hospital,Buraidah, Saudi Arabia
}

\begin{abstract}
Health care-associated bacteremia cause important morbidity and mortality and have a considerable impact on healthcare costs. Infections caused by extended spectrum beta-lactamase producing bacteria are of clinical and epidemiological importance, since their mobile genetic elements facilitate cross-infection. Objectives: To analyze health care-associated bacteremia in ICUs of King Fahad Specialized Hospital and to assess ESBL production in the isolated Gram negative bacteria. Methods: This study included 519 patients. Their blood samples were collected for blood culture. The isolates were identified and antibiotic sensitivity tests were performed. The type of B-lactamase gene was determined by polymerase chain reaction (PCR). Results: The rate of health care-associated bacteremia was 9.8\%. Gram positive organisms were detected in $67.8 \%$; methithilin resistant Staph.aureus (MRSA) was the most prevalent (17.8\%). Gram negative bacilli were detected in $30.6 \%$. E.coli was the most common $(12.9 \%)$. The production of extended spectrum $\beta$-lactamase (ESBL) en zy me was positive in $84.2 \%$ of the is olated Gram negative isolates. Temoniera (TEM) was the main type of $\beta$-lactamase. Conclusion: The isolation of multi-drug resistant bacteria and ESBL producing Gram negative organisms in ICU patients resulted in a greater awareness of implementation of new ru les for microbiological screening and infection control measures.
\end{abstract}

Keywords Health Care-Associated Bacteremia, Klebsiella Pneumoniae, Methicillin Resistant Staph Aureus, Extended Spectrum Beta-lactamase

\section{Introduction}

Health care-associated infections (HAI) cause important morbidity and mortality and have a considerable impact on healthcare costs. Effective infection control programs, such as surveillance, can reduce the infection rate by up to $32 \%[1]$.

Health care-associated blood-stream infections (BSI) create a serious health problem in hospitals all over the world. In addition, patients admitted to intensive care units (ICUs) carry an even higher risk of BSI than those admitted to other types of units (non- ICU) and data from the surveillance and control of pathogens of epidemiologic importance in United States hospitals showed that $49.4 \%$ of all BSI occurred in the ICU[2]. HAI are frequently associated with drug-resistant microorganis ms, including methicillin resistant Staphylococcus aureus (MRSA) and extended spectrum $\beta$-lactamase (ESBL)-producing Gram

* Corresponding author:

marwa75 jan@yahoo.com (Marwa Salah Mostafa)

Published online at http://journal.sapub.org/microbiology

Copyright (C) 2012 Scientific \& Academic Publishing. All Rights Reserved negative bacteria, which can pose considerable therapeutic problems [3].

Antimicrobial resistance is an increasing threat in hospitalized patients, and both mortality and morbidity from infections are greater when caused by antimicrobialresistant bacteria[4]. It is also noted that there is an increase in the resistance among Gram negative bacilli to third generation cephalosporins which is caused by expression of ESBL enzy mes.

ESBLs are enzymes that mediate resistance to extended-spectrum (third generation) cephalosporins (e.g., ceftazidime, cefotaxime, and ceftriaxone) and monobactams (e.g., aztreonam) but do not affect cephamycins (e.g., cefo xit in and cefotetan) or carbapenems (e.g., meropenem or imipenem) [5-7].

The aim of the present study is to identify health care-associated bacteremia in the ICUs and to describe the resistance patterns of the isolates especially those caused by ESBLs. The study also aimed to determine the types of $\beta$-lactamase genes responsible for resistance among $K$ pneumoniae isolates by polymerase chain reaction because $K$ pneumoniae bacteremia is a very important cause of morbidity and mortality in Gram negative bacteremia in 
most medically well-developed countries.

\section{Methodology}

\subsection{Study Design and Subjects}

The present study was conducted over a period of 12 months from May 2011 to April 2012 on patients admitted to different ICUs at KFSH. The total number of patients included in the study was 519 patients. They were monitored daily by the attending physicians for subsequent development of health care-as sociated bacteremia.

\subsection{Sample Collection and Processing}

Blood samples for culture were collected before the start of antibiotic therapy. The samples were inoculated immed iately under complete aseptic conditions into bottles containing $50 \mathrm{ml}$ brain heart infusion broth. The bottles were incubated aerobically at $37^{\circ} \mathrm{C}$ for 7 days and were examined daily for evidence of bacterial growth. Subcultures were done on blood agar.

\subsection{Identification of $B$ acterial Is ol ates}

Isolated colonies were evaluated according to their morphology, motility, Gram staining characteristics, catalase oxidase, coagulase tests, PYR test and latex agglutination test for Streptococci identification (Omega Diagnostica, Scotland, UK). Gram negative bacilli were further identified by API 20E system (Bomeriux SA, Monlien Vercica and France). Wet mount preparations were used to determine Candida species which was identified by germ tube test.

\subsection{Detection of Oxacillin Resistance}

Detection of oxacillin resistance was made by using Oxacillin Resistant Screening Agar Base[8].

\subsection{Anti microbi al Susceptibility}

Susceptibility was tested by modified Kirby-Bauer disc diffusion method using 0.5 McFarland turbidity of each isolate[9].

\subsection{Detection of $\beta$-Lactamase Production}

Beta-lactamase enzyme is detected among the Gram negative bacilli by the chromogenic method (Nitrocefin test- Oxoid, UK).

\subsection{Phenotypic Detection of Extended S pectrum $\beta$-Lactamases}

This was done by the combined disc method and E-test using Cefotaxime/cefotaxime + clavulanic acid (CT/ CTL) and Ceftazidime/ceftazidime + clavulanic acid (TZ/TZL) (AB BIODISK, Solna, Sweden)[10].

\subsection{Genotypic Detection and Deter mination of the Type} of $\beta$-Lactamase
Isolated strains of Klebsiella pneumoniae were investigated to determine the probable type of $\beta$-lactamase enzy me. The is olates were tested for TEM, SHV, CTX-M-1 and TOHO-1 genes by using PCR. DNA was extracted from clinical isolates and controls according to the method described by[11]. PCR reaction was performed in a final volume of $50 \mu 1$ containing $0.2 \mu \mathrm{M}$ of each primer, $200 \mu \mathrm{M}$ of each dNTPs, 1x reaction buffer, 2.5 units of Taq polymerase and $5 \mu \mathrm{M}$ of the template DNA. Klebsiella strains with known ESBL types: TEM, SHV, CTX-M-1 and TOHO-1 were included as positive controls. A reagent blank containing all components of the reaction mixture except template DNA (substituted with sterile distilled water) was included in PCR reaction as a negative control. The amplification conditions were as follows: $94^{\circ} \mathrm{C}$ for $5 \mathrm{~min}$; then 35 cycles at $94^{\circ} \mathrm{C}$ for $30 \mathrm{~s}, 58^{\circ} \mathrm{C}$ for $30 \mathrm{~s}$, and $72^{\circ} \mathrm{C}$ for $2 \mathrm{~min}$; and, finally, one cycle at $72{ }^{\circ} \mathrm{C}$ for 15 min. [12-14]. Primers sequences for are shown in table (1).

Table 1. Primer Sequences for Detection of $\beta$-Lactamases Genes

\begin{tabular}{|c|c|c|}
\hline Gene & Primers sequences & $\begin{array}{c}\text { Product } \\
\text { size } \\
\text { (bp) }\end{array}$ \\
\hline CTXM-1 & $\begin{array}{c}\text { F:5'-GACGAT GT CACT GGCT GAGC- 3' } \\
\text { R:5'-AGCCGCCGACGCT AAT ACA-3' }\end{array}$ & 499 \\
\hline TEM & $\begin{array}{c}\text { F:5'-TT GGGT GCACGAGT GGGTT A -3' } \\
\text { R:5'-TAATT GTT GCCGGGAAGCT A -3' }\end{array}$ & 503 \\
\hline SHV & $\begin{array}{c}\text { F:5'-TCGGGCCGCGT AGGCAT GAT -3' } \\
\text { R:5'-AGCAGGGCGACAAT CCCGCG -3' }\end{array}$ & 625 \\
\hline TOHO-1 & $\begin{array}{c}\text { F:5'-GCGACCTGGTTAACT ACAATCC-3' } \\
\text { R:5'-CGGTAGTATT GCCCTTAAGCC-3' }\end{array}$ & 351 \\
\hline
\end{tabular}

\section{Results}

A total of 51 patients developed health care-associated BSI $(9.8 \%)$. The age of the patients ranged between 5 and 75 years with a mean age of $42.4 \pm 16.7$ years. Nu mber of males was 36 out of $51(70.6 \%)$

The rate of bacteremia among different intensive care units (ICUs) is illustrated in table (2) which shows that the highest rate was in the chest ICU $(19.5 \%)$ followed by the trauma care unit (12.5\%) then the general ICU $(9.9 \%)$.

Out of 51 BSI patients, 45 patients had monomicrobial infection (88\%) and 6 had polymicrobial infection (12\%). Among these infections, 62 different microorganis ms were isolated. Analysis of these microorganis $m$ showed that Gram positive bacteria were reported in 42 isolates $(67.8 \%)$ while Gram negative bacteria were reported in 19 isolates (30.6\%). MRSA was isolated from 11/62 (17.8\%), followed by methicillin resistant coagulase negative Staphylococci 10 (16\%), E coli 8 (12.9\%), Klebsiella pneumoniae 6 (9.7\%), Candida spp. in one isolate (1.6\%) (Table 3).

Antibiotic sensitivity tests of Gram positive organisms showed that the isolated organisms were mostly sensitive to vancomycin (85.7\%), amikacin (47.6\%) and chloramphenicol (42.9\%). While, Gram negative organisms were mostly sensitive to imipenem $(89.4 \%)$, amikacin $(42.1 \%)$ and gentamic in $(31.5 \%)$. 
Table 2. Bacteremia among Different ICUs

\begin{tabular}{|c|c|c|c|}
\hline ICU & Frequence & Rate & Proport ion \\
\hline Trauma $(\mathrm{n}=80)$ & 10 & 12.5 & 19.6 \\
\hline Post Operative $(\mathrm{n}=26)$ & 2 & 7.7 & 3.9 \\
\hline Chest $(\mathrm{n}=87)$ & 17 & 19.5 & 33.3 \\
\hline Medicine $(\mathrm{n}=92)$ & 3 & 3.3 & 5.9 \\
\hline General $(\mathrm{n}=81)$ & 8 & 9.9 & 15.7 \\
\hline Coronary $(\mathrm{n}=83)$ & 6 & 7.2 & 11.8 \\
\hline Tropical $(\mathrm{n}=70)$ & 5 & 7.1 & 9.8 \\
\hline Total $(\mathrm{n}=519)$ & 51 & 9.8 & 100.0 \\
\hline
\end{tabular}

*(Rate is calculated according to the number of patients admitted to each ICU)

**(Proportion is calculated according to the total number of infected patients)

Table 3. Distribution of Pathogens Associated with NBSI

\begin{tabular}{|c|c|}
\hline Pathogens & No $(\%) \mathrm{n}=62$ \\
\hline Gram-Positive Organisms & $42(67.8)$ \\
MRSA & 11 \\
MRCoNS & 10 \\
MSSA & 8 \\
S.pneuminae & 5 \\
Enterococcus spp. & 5 \\
Viridans streptococci & 3 \\
\hline Gram-Negative Organisms & $19(30.6)$ \\
E.coli & 8 \\
K.pneumoniae & 6 \\
Enterobacter species & 1 \\
Acinetobacter baumannii & 2 \\
Pseudomonas aeruginosa & $1(1.6)$ \\
\hline Candida spp. &
\end{tabular}

\subsection{Analysis of $\beta$-lactamase producers}

Out of 62 organis ms causing BSI, $19(30.6 \%)$ were Gram negative bacilli (table 4). All these strains were screened for the production of $\beta$-lactamase enzyme by nitrocefin test, which indicated that $16 / 19(84.2 \%)$ were $\beta$-lactamase producers with the largest percent $(100 \%)$ being in $E$ coli,
Pseudomonas aeruginosa and Acinetobacter baumannii.

Table 4. Gram Negative Bacilli Isolated from BSI and $\beta$-Lactamase Production

\begin{tabular}{|c|c|c|}
\hline Microorganism & $\begin{array}{c}\text { No }(\%) \\
\mathrm{n}=19\end{array}$ & $\begin{array}{c}\beta \text {-Lactamse } \\
\text { Production }\end{array}$ \\
\hline E coli & $8(42.1 \%)$ & $8(100 \%)$ \\
\hline Klebsiella pneumoniae & $6(31.5 \%)$ & $4(66.7 \%)$ \\
\hline Enterobacter spp. & $2(10.5 \%)$ & $1(50 \%)$ \\
\hline $\begin{array}{c}\text { Acinetobacter } \\
\text { baumannii }\end{array}$ & $1(5.4 \%)$ & $1(100 \%)$ \\
\hline $\begin{array}{c}\text { Pseudomonas } \\
\text { aeruginosa }\end{array}$ & $2(10.5 \%)$ & $2(100 \%)$ \\
\hline $\begin{array}{c}\text { Total Gram Negative } \\
\text { Bacilli }\end{array}$ & 19 & $16 / 19(84.2 \%)$ \\
\hline
\end{tabular}

\subsection{Analysis of ESB L production by confirmatory tests among Gram negative bacilli}

Confirmatory tests for ESBL production including Combined Disk method and ESBL E-Test revealed that $12 / 19(63.2 \%)$ of isolates was confirmed to be ESBL producers (Table 5). ESBL production was confirmed in $6 / 8$ E coli strains $(75 \%), 4 / 6 \mathrm{~K}$ pneumoniae $(66.7 \%), 1 / 2$ Enterobacter species $(50 \%)$ and $1 / 1$ Acinetobacter baumanii (100\%).

\subsection{Deter mination of the type of $\beta$-lactamase by PCR in K pneumoniae isolates}

Types of $\beta$-lactamase results are shown in table (6). It can be noticed that TEM was the main type of $\beta$-lactamase, SHV was the second, followed by CTX-M1, no TOHO-1 genes were detected in the tested isolates.

Table 5. Preliminary screening and confirmatory tests for ESBL among Gram negative organisms isolated from patients

\begin{tabular}{|c|c|c|c|}
\hline \multirow{2}{*}{$\begin{array}{c}\text { Organisms } \\
(\mathrm{n}=19)\end{array}$} & \multirow{2}{*}{ Screening } & \multicolumn{2}{|c|}{ Confirmatory tests } \\
\cline { 3 - 4 } & & Combined disk method & \multirow{2}{*}{ ESBL-E Test } \\
\hline E coli 8 & $8(100 \%)$ & $6(75 \%)$ & $6(75 \%)$ \\
\hline Kneumoniae 6 & $4(66.7 \%)$ & $4(66.7 \%)$ & $4(66.7 \%)$ \\
\hline Enterobacter spp. & $1(50 \%)$ & $1(50 \%)$ & $1(50 \%)$ \\
\hline Acinetobacter baumannii 1 & $1(100 \%)$ & $1(100 \%)$ & $0(0 \%)$ \\
\hline Pseudomonas aeruginosa 2 & $2(100 \%)$ & $0(0 \%)$ & $12(63.2 \%)$ \\
\hline Total 19(100\%) & $16(84.2 \%)$ & $12(63.2 \%)$ & \\
\hline
\end{tabular}

Table 6. Distribution of $\beta$-Lactamase Genes among Kpneumoniae strains

\begin{tabular}{|c|c|}
\hline Gene Type & Klebsiella pneumoniae $(\mathrm{n}=6)$ \\
\hline TEM & $5(83.3 \%)$ \\
\hline SHV & $4(66.7 \%)$ \\
\hline CTX-M-1 & $3(50 \%)$ \\
\hline TOHO-1 & $0(0 \%)$ \\
\hline TEM+SHV & $3(50 \%)$ \\
\hline TEM+CTX-M-1 & $3(50 \%)$ \\
\hline TEM+SHV+CTX-M-1 & $1(16.7 \%)$ \\
\hline
\end{tabular}




\subsection{ESBLProduction by Phenotypic Methods and Correlation with PCR}

Out of 6 isolates of $K$ pneumoniae strains, 5 strains $(83.3 \%)$ could be identified as ESBL producers by PCR, 4 of them $(66.7 \%)$ could be identified as ESBL producers by Combined Disk method and ESBL E-Test, the strain which was negative by phenotypic methods showed TEM enzyme by PCR.

\section{Discussion}

NBSI create a serious health problem in hospitals all over the world. They contribute to greater morbidity and mortality rates, as well as to increasing length of hospital stay and health care cost[3]. In the present study, the rate of health care-associated BSI was $9.8 \%$. This result was higher than those reported by [15] in Canada $(0.69 \%)$.

The high rate of health care associated BSI in this study may be due to the high risk patients enrolled in the study and the presence of several risk factors associated with those patients.

In this study we tried to assess the risk factors associated with BSI. There was a higher significant difference in patients with previous prescription of antibiotics who developed BSI $(34 / 51,66.7 \%)$ compared to $216 / 468$ of patients without BSI $(46.2 \%)(P=0.02)$, there was also a higher significant difference in patients on mechanical ventilation who developed BSI $(18 / 51,35.3 \%)$ co mpared to $102 / 468$ of patients without BSI $(21.8 \%)(P=0.03)$. There was also higher percentage of patients with urinary catheterization who developed BSI 20/51 (39.2\%) compared to $129 / 468$ of patients who had no BSI $(27.6 \%)$, but with non-significant difference $(P=0.081)$, This is supported by the results of another study[2] who reported that risk factors for acquisition of BSI in the ICU included previous prescription of antibiotics, mechanical ventilation and the use of nasogastric tube.

Our results were in concordance with surveillance for nosocomial bloodstream infections at 49 hospitals over a 3 -year period detected $>10,000$ infections. Gram positive organisms accounted for $64 \%$ of cases, Gram negative organisms accounted for 27\%. The most common organisms were coagulase negative staphylococci $(32 \%)$, Staphylococcus aureus (16\%)[16].

In our study, we noticed that MRSA strains had high overall resistance to penicillin $(100 \%)$, erythromycin (90.9\%), clindamycin (81.8\%), ampicillin (100\%), ciprofloxacin $(72.7 \%)$, tetracycline $(63.6 \%)$, cefotaxime $(81.8 \%)$, gentamicin $(63.6 \%)$ and amoxicillin/clavulan ic $(90.9 \%)$. These results agreed with[8], who found that resistance rates of MRSA were as follows: ciprofloxac in $(89 \%)$, clindamycin and erythromycin ( $94 \%$ for each), tetracycline $(33 \%)$.

In our study, few isolates $(9.1 \%)$ were found to be vancomycin resistant. This report paralleled what is reported by different studies which indicated that vancomycin intermed iate $S$. aureus strains are beginning to emerge[4].

IN the present study, Gram negative organis ms were mostly sensitive to imipenem (89.4\%), amikacin $(42.1 \%)$ and gentamicin (31.5\%), these results are similar to[17] who reported $47.7 \%$ resistance to amikac in and $81 \%$ resistance to gentamycin.

Kanamori et al[18] showed that $71.4 \%$ of the isolates were resistant to gentamycin, while all of the ESBL-producing isolates were susceptible to imepenem. It has been reported that the prevalence of cores istance to CIP, GEN, and SXT was high among phenotypically identified ESBL-producing E. coli and $K$. pneumoniae in the Philippines[19]. Imipenem remains fully effective against ESBL producers and carbapenems may be the most appropriate agents for severe infections due to ESBL-producing Enterobacteriaceae[14],[17-18].

In the present study, a total of $63.2 \%(12 / 19)$ of isolates were confirmed to be ESBL producers. These results are in concordance to[14] who found $73.2 \%$ ESBL phenotypic expression among Enterobacteria. Akujobi and Ewuru Chika et al[9] reported ESBL production in $11.4 \%$ of Klebsiella isolates. A study confirmed ESBL production in $17 / 91$ (18.7\%) of strains of Enterobacteriacae and among all of the 3 klebsiella species tested[18]. Another study reported ESBL production in 65/207 (31.4\%) of Klebsiella, Enterobacter and Serratia strains with a predominance of Klebsiella pneumoniae (29/65)[19]. The resistance phenomenon is on the increase, this increasing resistance is due to inappropriate usage of antimicrobial drugs such as overuse, misuse, suboptimal dosage and non-compliance with treatment duration[9].

In the present study, the type of $\beta$-lactamase gene was determined among $K$ pneumoniae strains by PCR in which TEM was the main type of $\beta$-lactamase, followed by SHV, then CTX-M1. Some $K$ pneumoniae strains produced more than one type of $\beta$-lactamase. Vasques et al[14] reported TEM in 13/24, CTX-M 1 in 7/24 and SHV in 6/24 of ESBL producing Enterobacteria and they also reported that Klebsiella and E coli predominated in the ESBL producing species identified. Predominance of SHV in 13/14 of $K$ pneumoniae strains followed by TEM and CTX-M (9/14 for each of them) was reported[17]. Several studies showed expression of more than one ESBL genes which is in concordance to the present study[14],[17],[20].

\section{Conclusions}

The isolation of multi-drug resistant bacteria and ESBL producing Gram negative organisms in ICU patients during the study period resulted in a greater awareness to the presence of multi-drug resistance among bacterial isolates in our hospital ICU units. New rules for microbiological screening and infection control measures are recommended i.e. testing for beta-lactamase production and isolation precautions for patients in whom these microorganis ms are identified. In addition, restriction of the use of 
third-generation cephalosporins, along with care in the use and abuse of antimicrobials to minimize the emergence of resistant strains, are the most effective strategies for controlling and decreasing blood stream in fections and the spread of ESBL-producing and multi-drug resistant organisms.

\section{ACKNOWLEDGEMENTS}

We gratefully acknowledge the deanship of scientific research, Qassim University that solely funded this study.

\section{REFERENCES}

[1] F.S. Erdinc, M.A. Yetkin, C. Ataman Hatipoglu, M. Yucel, A.E. Karakoc, M.A. Cevik, N. Tulek, "Five-year surveillance of nosocomial infections in Ankara Training and Research Hospital", Elsevier, Journal of Hospital Infection, vol.64, pp.391-396, 2006.

[2] Vesna Šuljagić, Miloje Čobeljić, Slavenka Janković, Veljko Mirović, Ljiljana Marković-Denić,Predrag Romić, Dragan Mikić, "Nosocomial bloodstream infections in ICU and non-ICU patients", Elsevier, American Journal of Infection Control, vol.33, pp.333-340, 2005.

[3] Jason W. Bennett, Clinton K. Murray, Robert L. Holmes, Jan E. Patterson, James H. Jorgensen,, "Diminished vancomycin \& daptomycin susceptibility during prolonged bacteremia with MRSA", Elsevier, Diagnostic Microbiology \& Infectious Disease, vol.60, pp.437-440, 2008.

[4] Ahmed S. Al-Dhaheri, Mohamed S. Al-Niyadi, Ahmed D. Al-Dhaheri, Salim M. Bastaki, "Resistance patterns of bacterial isolates to antimicrobials from 3 hospitals in the UAE", Saudi Medical Journal, vol.30, no.5, 618-623, 2009.

[5] Mark E. Rupp, Paul D Fey, Rupp ME, Fey PD, "Extended-spectrum $\beta$-lactamase (ESBL) - producing Enterobacteriaceae: Considerations for diagnosis, prevention and drug treatment", Adis, Drugs, vol.63, pp.353-365, 2003.

[6] Johann DD Pitout, Kevin B Laupland, "Extended-spectrum â-lactamase-producing Enterobacteriaceae: an emerging public-health concern”, Elsevier, Lancet Infectious Diseases., vol.8, pp.159-166, 2008.

[7] Karen Bush, "Bench-to-bedside review: the role of â-lactamases in antibioticresistant gram-negative infections", BioMed Central, Critical Care, vol.14, pp.224, 2010.

[8] Simor A.E, Ofner-Agostini M, Bryce E, Green K, McGeer A, Mulvey M, Paton S, "The evaluation of methicillin resistant Staphylococcus aureus in Canadian hospitals: 5 years of national surveillance", Canadian Medical Association, Canadian Medical Association Journal, vol.165 no.1, pp.21-26, 2001.

[9] Akujobi C.N. and Ewuru Chika P., "Detection of extended spectrum $\beta$-lactamase in Gram negative bacilli from clinical specimens in a teaching hospital in South Eastern Nigeria", Nigerian Medical Journal, vol.5, no.4, pp.141-146, 2010.

[10] National Committee for Clinical Laboratory Standards,
"Performance Standards for Antimicrobial Susceptibility Testing; Thirteenth Informational Supplement", NCCLS documents M 100-S13, Wayne, Pennsylvania, USA, 2003.

[11] Tobias Kieser, "Factors affecting the isolation of CCC DNA from Streptomyces lividans and Escherichia coli", Elsevier, Plasmid, vol.12, pp.19-36, 1984.

[12] Jesus Silva, Rodolfo Gstica, Cecilia Aguilar, Zita Becerra, Ulises Garza-Ramos, Manuel Velazquez, GuadalupeMiranda, Blanca Leanos, Fortino Solorzano, Gabriela Echaniz, "Outbreak of infection with extended-spectrum betalactamase producing Klebsiella pneumoniae in a Mexican hospital", American Society for Microbiology, Journal of Clinical Microbiology, vol.39, no.9, pp.3193-3196, 2001.

[13] Johann D.D. Pitout, Ashfaque Hossain, Nancy D. Hanson, "Phenotypic and molecular detection of CTX-M-betalactamases produced by Escherichia coli and Klebsiella spp.", American Society for Microbiology, Journal of Clinical Microbiology, vol.42, no.12, pp.5715-5721, 2004.

[14] Marcia Regina G. Vasques, Alexandre Ribeiro Bello, Cristiane da Cruz Lamas, Juarez Correa, Jose Augusto Adler Pereira, " $\beta$-lactamase producing enterobacteria isolated from surveillance swabs of patients in a cardiac intensive care unit in Rio de Janeiro, Brazil", Elsevier, Brazilian Journal of Infectious Diseases., vol.15, no.1, pp.28-33, 2011.

[15] Wendy Sligl, Geoffrey Taylor, Peter G. Brindley, "Five years of nosocomial Gram-negative bacteremia in a general intensive care unit: epidemiology, antimicrobial susceptibility patterns, and outcomes", Esevier, International society for infectious diseases, vol.10, pp.320-325, 2006.

[16] Michael B. Edmond, Sarah E. Wallace, Donna K. McClish, Michael A. Pfaller, Ronald N. Jones, and Richard P. Wenzel, Edmond MB, Wallace SE, McClish D.K., Pfaller MA, Jones $\mathrm{R}$ and Wenzel RP, "Nosocomial bloodstream infections in United States hospitals: a 3-year analysis", Infectious Diseases Society of America, Clinical Infectious Diseases, vol.29, pp.239-244, 1999.

[17] S. Nedjai, A. Barguigua, N. Djahmi, L. Jamali, K. Zerouali, M. Dekhil, M. Timinoun, "Prevalence and characterization of extended spectrum $\beta$-lactamases in KlebsiellaEnterobacter-Serratia group bacteria, in Algeria", Elsevier, Médecine et maladies infectieuses, vol.42, pp.20-29, 2012.

[18] Hajime Kanamori, Rizalina B. Navarro, Hisakazu Yano, Lydia T. Sombrero, Ma. Rosario Z. Capeding, Socorro P. Lupisan, Remigio M. Olveda, Kazuaki Arai, Hiroyuki Kunishimad, Yoichi Hirakatac, Mitsuo Kaku, "Molecular characteristics of extended-spectrum â-lactamases in clinical isolates of Enterobacteriaceae from the Philippines", Elsevier, Acta Tropica, vol.120 pp.140-145, 2011.

[19] Yoichi Hirakata, Junichi Matsuda, Yoshitsugu Miyazaki, Shimeru Kamihira, Sayoko Kawakami, Yukihisa Miyazawa, Yasuo Ono, Nobuhiko Nakazaki, Yasuyoshi Hirata, Matsuhisa Inoue, John D. Turnidge, Jan M. Bell, Ronald N. Jones, Shigeru Kohno, "Regional variation in the prevalence of extended-spectrum $\beta$-lactamase-producing clinical isolates in the Asia-Pacific region (SENTRY 1998-2002)", Elsevier, Diagnostic Microbiology \& Infectious Disease, vol.52, pp.323-329, 2005.

[20] George A. Jacoby,Luisa Silvia Munoz-Price, "The new beta-lactamases", New England Journal of Medicine, vol.352, no.4, pp.380-391, 2005. 